\title{
Commentary: Association Between Antihypertensive Medication Use and Breast Cancer: A Systematic Review and Meta-Analysis
}

\author{
Natansh D. Modi ${ }^{1}$, Ahmad Y. Abuhelwa ${ }^{1}$, Bradley D. Menz ${ }^{2}$, Ross A. McKinnon ${ }^{1}$, \\ Michael J. Sorich ${ }^{1}$ and Ashley M. Hopkins ${ }^{1 *}$ \\ ${ }^{1}$ College of Medicine and Public Health, Flinders University, Adelaide, SA, Australia, ${ }^{2}$ Division of Pharmacy, Southern Adelaide \\ Local Health Network, Flinders Medical Centre, Adelaide, SA, Australia
}

Keywords: commentary articles, concomitant, breast cancer, immunotherapies, beta-blocker

\section{OPEN ACCESS}

Edited by:

Marie-Odile Parat,

The University of Queensland,

Australia

Reviewed by:

Erica Sloan,

Monash University, Australia

*Correspondence:

Ashley M. Hopkins

ashley.hopkins@flinders.edu.au

Specialty section:

This article was submitted to Pharmacology of Anti-Cancer Drugs,

a section of the journal

Frontiers in Pharmacology

Received: 29 June 2021

Accepted: 21 July 2021

Published: 05 August 2021

Citation:

Modi ND, Abuhelwa AY, Menz BD,

McKinnon RA, Sorich MJ and

Hopkins AM (2021) Commentary:

Association Between Antihypertensive Medication Use and Breast Cancer: A Systematic Review and Meta-Analysis.

Front. Pharmacol. 12:732622.

doi: 10.3389/fphar.2021.732622

\section{A Commentary on}

Association Between Antihypertensive Medication Use and Breast Cancer: A Systematic Review and Meta-Analysis

by Xie, Y., Wang, M., Xu, P., Deng, Y., Zheng, Y., and Yang, S. (2021). Front. Pharmacol. 10:609901. doi: 10.3389/fphar.2021.609901

\section{INTRODUCTION}

In an emerging age of precision medicine, clarity on the impact of concomitant non-cancer medicines on the efficacy of anti-cancer treatments has never been so important. For example, immunotherapies are emerging as an important treatment option in breast cancer (e.g., atezolizumab, pembrolizumab) (Schmid et al., 2018; Cescon et al., 2020). Further, it is hypothesised that concomitant medicines such as beta-blockers, statins, and metformin may boost immunotherapy actions (Kokolus et al., 2017; Afzal et al., 2018; Cortellini et al., 2020), while antibiotics and proton pump inhibitors may impair responses (Hopkins et al., 2020a; Hopkins et al., 2020b; Cortellini et al., 2020). However, undertaking randomised control trials to identify the impact of each non-cancer concomitant medicine on breast cancer prognosis is not practicable due to cost, exposure to potentially harmful strategies, and years until results become apparent. Thus, we read with great interest the systematic review and meta-analysis from Xie et al. on the association between concomitant antihypertensive medication use and breast cancer risk and prognosis (breast cancerspecific mortality, recurrence, overall survival, and disease-specific survival) (Xie et al., 2021). Xie et al. (2021) concluded that the use of calcium channel blockers, beta-blockers, or diuretics was significantly associated with an increased risk of developing breast cancer, and that diuretic use may elevate the risk of breast cancer-specific mortality (with no statistically significant association found for calcium channel blockers, beta-blockers, or reninangiotensin system inhibitors). Underlying assumptions of these conclusions are that the meta-analysis strategy acquired sufficient power to assess the associations and the author team adequately evaluated and filtered original scientific literature for quality. In relation to this evaluation, we appreciate the scoring system used by the authors to differentiate the 
quality of prior studies, however greater detail on the specifics of each study achieving their score would be appreciated.

\section{TYPE OF DATA}

Evaluating the association between concomitant antihypertensive use and survival outcomes in breast cancer has been undertaken using multiple data types, including observational, health registry, electronic health record (EHR), and clinical trial data. Each data type has strengths and weaknesses for providing affordable, rapid, hypothesis-generating findings. For example, health registries and EHR are often very large and matched to contemporary practice, but may have limited value with regards to treatment, comorbidity, cancer subtype, and other adjustment data to extract causality insights (as compared to some trial datasets) (Mack et al., 2018). As such, it would be useful if Xie et al. (2021) provides clear information on the data types used in each metastudy and whether results differ according to registry/EHR versus clinical trial data.

\section{IMMORTAL TIME BIAS}

Immortal time bias may greatly inflate the apparent association between an exposure (e.g., beta-blocker use) and survival in observational studies that investigate an exposure that is not known at the start of the survival follow-up period (e.g., at cancer diagnosis) (Lévesque et al., 2010). If individuals who commence the use of a beta-blocker at a later time are included in the exposed group, then it is important to use more complex analysis methods (e.g., landmark, time-dependent covariate) to avoid immortal time bias (Weberpals et al., 2016).

We note that Cardwell et al. (2014) and Cui et al. (2019) provide explicit statements on their methodologies to account for immortal time bias (nested case-control and time-dependent approach respectively). We would appreciate it if Xie et al. (2021) could provide information on whether each study used methodologies to

\section{REFERENCES}

Afzal, M. Z., Mercado, R. R., and Shirai, K. (2018). Efficacy of Metformin in Combination with Immune Checkpoint Inhibitors (Anti-PD-1/anti-CTLA-4) in Metastatic Malignant Melanoma. J. ImmunoTherapy Cancer 6 (1), 64. doi:10.1186/s40425-018-0375-1

Cardwell, C. R., Coleman, H. G., Murray, L. J., Entschladen, F., and Powe, D. G. (2014). Beta-blocker Usage and Breast Cancer Survival: a Nested Case-Control Study within a UK Clinical Practice Research Datalink Cohort. Int. J. Epidemiol. 42 (6), 1852-1861. doi:10.1093/ije/dyt196

Cescon, D. W., Nowecki, Z., Gallardo, C., Holgado, E., Iwata, H., Masuda, N., et al. (2020). Pembrolizumab Plus Chemotherapy versus Placebo Plus Chemotherapy for Previously Untreated Locally Recurrent Inoperable or Metastatic Triple-Negative Breast Cancer (KEYNOTE-355): a Randomised, Placebo-Controlled, Double-Blind, Phase 3 Clinical Trial. Lancet 396 (10265), 1817-1828.

Cortellini, A., Tucci, M., Adamo, V., Stucci, L. S., Russo, A., Tanda, E. T., et al. (2020). Integrated Analysis of Concomitant Medications and mitigate immortal time bias, and whether meta-analysis results differed between studies that did and did not account for immortal time bias.

\section{INFLUENCE OF STAGE, SUBTYPES, AND THERAPY}

We advocate that analyses performed to identify the association between antihypertensive use and survival outcomes in breast cancer need to differentiate between subtypes (e.g., HER2 positive, triple-negative), stages of disease (early vs. advanced), and lines of therapy. For example, Modi et al. (2020) identified worse overall survival amongst patients using beta-blockers and treated with contemporary trastuzumab, pertuzumab, ado-trastuzumab emtansine, and docetaxel therapies for HER2 positive advanced disease. In contrast, Spera et al. (2017) identified improved progressionfree survival amongst patients using beta-blockers and treated with contemporary ramucirumab and docetaxel therapies for HER2 negative advanced disease.

These opposing results demonstrate findings may be biologically different between breast cancer subtypes, stages, lines of therapy, or treatment options and that future evaluation of the associations between antihypertensive use and outcomes in subgroups would be valuable.

\section{AUTHOR CONTRIBUTIONS}

All authors were involved in data analyses and writing the manuscript.

\section{FUNDING}

RM and MS are supported by Beat Cancer Research Fellowships from Cancer Council South Australia. NM is supported by the NHMRC Postgraduate scholarship, Australia (APP2005294).

Oncological Outcomes from PD-1/pd-L1 Checkpoint Inhibitors in Clinical Practice. J. ImmunoTherapy Cancer 8 (2), e001361. doi:10.1136/jitc-2020-001361

Cui, Y., Wen, W., Zheng, T., Li, H., Gao, Y.-T., Cai, H., et al. (2019). Use of Antihypertensive Medications and Survival Rates for Breast, Colorectal, Lung, or Stomach Cancer. Am. J. Epidemiol. 188 (8), 1512-1528. doi:10.1093/aje/ kwz106

Hopkins, A. M., Kichenadasse, G., Karapetis, C. S., Rowland, A., and Sorich, M. J. (2020). Concomitant Antibiotic Use and Survival in Urothelial Carcinoma Treated with Atezolizumab. Eur. Urol. 78 (4), 540-543. doi:10.1016/ j.eururo.2020.06.061

Hopkins, A. M., Kichenadasse, G., Karapetis, C. S., Rowland, A., and Sorich, M. J. (2020). Concomitant Proton Pump Inhibitor Use and Survival in Urothelial Carcinoma Treated with Atezolizumab. Clin. Cancer Res. 26 (20), 5487-5493. doi:10.1158/1078-0432.ccr-20-1876

Kokolus, K. M., Zhang, Y., Sivik, J. M., Schmeck, C., Zhu, J., Repasky, E. A., et al. (2017). Beta Blocker Use Correlates with Better Overall Survival in Metastatic Melanoma Patients and Improves the Efficacy of Immunotherapies in Mice. Oncoimmunology 7 (3), e1405205-e. doi:10.1080/2162402x.2017.1405205 
Lévesque, L. E., Hanley, J. A., Kezouh, A., and Suissa, S. (2010). Problem of Immortal Time Bias in Cohort Studies: Example Using Statins for Preventing Progression of Diabetes. BMJ 340, b5087. doi:10.1136/bmj.b5087

Mack, C., Su, Z., and Westreich, D. (2018). AHRQ Methods for Effective Health Care. Managing Missing Data in Patient Registries: Addendum to Registries for Evaluating Patient Outcomes: A User's Guide. Third Edition. Rockville (MD): Agency for Healthcare Research and Quality (US). doi:10.23970/ ahrqregistriesmissingdata

Modi, N. D., Tan, J. Q. E., Rowland, A., Koczwara, B., Kichenadasse, G., McKinnon, R. A., et al. The Influence of Pre-existing Beta-Blockers Use on Survival Outcomes in HER2 Positive Advanced Breast Cancer: Pooled Analysis of Clinical Trial Data. 2020;10(1130).doi:10.3389/fonc.2020.01130

Schmid, P., Adams, S., Rugo, H. S., Schneeweiss, A., Barrios, C. H., Iwata, H., et al. (2018). Atezolizumab and Nab-Paclitaxel in Advanced Triple-Negative Breast Cancer. N. Engl. J. Med. 379 (22), 2108-2121. doi:10.1056/ nejmoa1809615

Spera, G., Fresco, R., Fung, H., Dyck, J. R. B., Pituskin, E., Paterson, I., et al. (2017). Beta Blockers and Improved Progression-free Survival in Patients with Advanced HER2 Negative Breast Cancer: a Retrospective Analysis of the ROSE/TRIO-012 Study. Ann. Oncol. 28 (8), 1836-1841. doi:10.1093/annonc/ mdx264

Weberpals, J., Jansen, L., Carr, P. R., Hoffmeister, M., and Brenner, H. (2016). Beta Blockers and Cancer Prognosis - the Role of Immortal Time Bias: A Systematic
Review and Meta-Analysis. Cancer Treat. Rev. 47, 1-11. doi:10.1016/ j.ctrv.2016.04.004

Xie, Y., Wang, M., Xu, P., Deng, Y., Zheng, Y., Yang, S., et al. Association between Antihypertensive Medication Use and Breast Cancer: A Systematic Review and Meta-Analysis. 2021;12(1169).doi:10.3389/fphar.2021.609901

Conflict of Interest: The authors declare that the research was conducted in the absence of any commercial or financial relationships that could be construed as a potential conflict of interest.

Publisher's Note: All claims expressed in this article are solely those of the authors and do not necessarily represent those of their affiliated organizations, or those of the publisher, the editors and the reviewers. Any product that may be evaluated in this article, or claim that may be made by its manufacturer, is not guaranteed or endorsed by the publisher.

Copyright (c) 2021 Modi, Abuhelwa, Menz, McKinnon, Sorich and Hopkins. This is an open-access article distributed under the terms of the Creative Commons Attribution License (CC BY). The use, distribution or reproduction in other forums is permitted, provided the original author(s) and the copyright owner(s) are credited and that the original publication in this journal is cited, in accordance with accepted academic practice. No use, distribution or reproduction is permitted which does not comply with these terms. 